\title{
An experimental study on the molecular organization and exciton diffusion in a bilayer of a porphyrin and poly(3-hexylthiophene)
}

\author{
Annemarie Huijser, ${ }^{\text {a) }}$ Tom J. Savenije, ${ }^{\text {b) }}$ Avi Shalav, ${ }^{\text {c) }}$ and Laurens D. A. Siebbeles \\ Opto-Electronic Materials Section, DelftChemTech, Delft University of Technology, Julianalaan 136, \\ 2628 BL Delft, The Netherlands
}

(Received 18 September 2007; accepted 27 May 2008; published online 6 August 2008)

\begin{abstract}
The exciton root-mean-square displacement $\left(\Lambda_{D}\right)$ in regioregular poly(3-hexylthiophene) (P3HT) deposited onto meso-tetrakis (n-methyl-4-pyridyl) porphyrin tetrachloride $\left(\mathrm{H}_{2} \mathrm{TMPyP}\right)$ has been determined from the photovoltaic response of a device based on these materials in a bilayer configuration. Excitons formed on illumination that reach the interface between $\mathrm{H}_{2} \mathrm{TMPyP}$ and P3HT can undergo interfacial charge separation by electron injection into the $\mathrm{H}_{2} \mathrm{TMPyP}$ and hole injection into the P3HT. The incident photon to current efficiency (IPCE) exceeds $20 \%$ over a broad wavelength regime. The theoretical analysis of the IPCE values gives a value for $\Lambda_{D}$ in $\mathrm{H}_{2}$ TMPyP that amounts to $14 \mathrm{~nm}$, while for P3HT a value of $18 \mathrm{~nm}$ is obtained. The latter value exceeds literature values reported for P3HT by almost a factor of 3. X-ray diffraction analysis shows that in the studied bilayer the P3HT backbones are aligned parallel to the interface with $\mathrm{H}_{2}$ TMPyP. In contrast, in the case of P3HT deposited onto $\mathrm{TiO}_{2}$, for which $\Lambda_{D}$ has been reported to equal only 7 $\mathrm{nm}$, hardly any organization of the P3HT backbones is observed. The excitonic coupling between P3HT backbones deposited onto $\mathrm{H}_{2}$ TMPyP is as high as $125 \mathrm{~cm}^{-1}$, a factor of 3 larger than the excitonic coupling between the disordered P3HT backbones that amounts to $47 \mathrm{~cm}^{-1}$. The difference illustrates the importance of controlling the molecular organization for the realization of efficient energy transfer in organic optoelectronics. (C) 2008 American Institute of Physics.
\end{abstract}

[DOI: $10.1063 / 1.2958325$ ]

\section{INTRODUCTION}

Conjugated electron-donating polymers such as substituted poly(phenylene-vinylene) (PPV) and polythiophene derivatives are considered as attractive photoactive materials because of their strong absorption band in the visible and their ease of processing into thin films. The binding energy of an exciton, formed on absorption of a photon, ranges typically from 0.2 to $1.0 \mathrm{eV}^{1-3}$ In order to realize an efficient photovoltaic device, excitons have to be dissociated into charges. This can be accomplished at an interface with an electron-accepting material. The driving force for charge separation has to exceed the exciton binding energy to allow exciton dissociation into charges. Electron-accepting materials studied intensively in combination with conjugated polymers include $\mathrm{TiO}_{2},{ }^{4,5}$ perylene derivatives, ${ }^{6}$ and fullerenes. Initially, bilayers of electron donor and electron acceptor were investigated. An important parameter in such organicbased solar cells is the average distance excitons are able to cover by diffusion before decay to the ground state occurs, referred to as the exciton root-mean-square displacement $\left(\Lambda_{D}\right)$. In the case of one-dimensional exciton diffusion, $\Lambda_{D}$ is defined by

\footnotetext{
${ }^{a)}$ Present address: Department of Chemical Physics, Lund University, Box 124, S-22100 Lund, Sweden.

${ }^{b)}$ Electronic mail: t.j.savenije@tudelft.nl.

${ }^{c}$ Present address: Department of Electronic Materials Engineering, The Research School of Physical Sciences and Engineering, Building 60, ANU Campus, Canberra, ACT 0200, Australia.
}

$$
\Lambda_{D}=\sqrt{2 D_{E} \tau_{E}},
$$

with $D_{E}$ the exciton diffusion coefficient and $\tau_{E}$ the exciton lifetime. Note that the degree of exciton diffusion is usually characterized by the exciton diffusion length $\left(\Lambda_{E}\right)$, as follows:

$$
\Lambda_{E}=\sqrt{D_{E} \tau_{E}} .
$$

Characterizing the extent of exciton diffusion by $\Lambda_{D}$ is, however, more useful, since Eq. (2) does not take into account the dimensionality of the exciton diffusion process and does not represent the average distance an exciton is able to cover by diffusion. The low efficiencies observed for organic bilayer devices have been attributed to the discrepancy in $\Lambda_{D}$ of typically only a few nanometers ${ }^{8-12}$ and the optical absorption length in the order of $100 \mathrm{~nm}$. To overcome this limitation, the bulk-heterojunction cell has been developed, which is based on an interpenetrating network of electron donor and electron acceptor. ${ }^{13,14}$ To date, a maximum performance of $4.4 \%$ has been realized for a photovoltaic cell based on a blend of poly(3-hexylthiophene) and a fullerene derivative. ${ }^{15,16}$ For a bulk heterojunction, it is essential to dissociate as much as possible the excitons formed on light absorption into charge carriers. To achieve this, the size of each phase in a blend should not exceed $\Lambda_{D}$. In addition, the morphology of the interpenetrating network should allow efficient charge transport. ${ }^{17-20}$ A particular blend morphology, with specific domain dimensions, is not necessarily optimal for all these independent processes. However, enhancement of the exciton root-mean-square displacement allows the use 

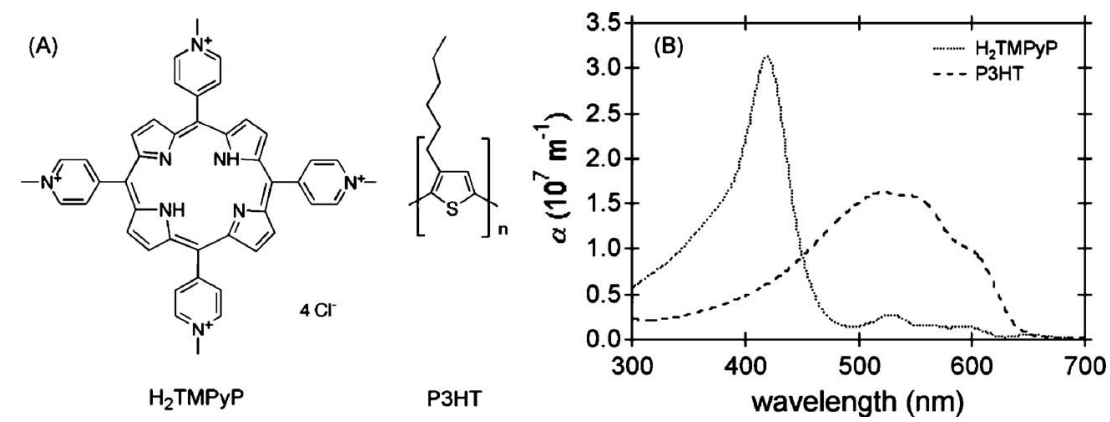

FIG. 1. Chemical structures (a) and the exponential optical absorption coefficient as function of wavelength (b) of $\mathrm{H}_{2}$ TMPyP and P3HT.

of larger domains of electron donor and electron acceptor, which could enhance transport of charge carriers from the exciton dissociating interface through the interpenetrating network toward the electrodes. ${ }^{21,22}$ A value for $\Lambda_{D}$ in the same order of magnitude as the optical absorption length would make the application of the electron donor and electon acceptor in a bulk heterojunction obsolete.

Important advantages of alkyl substituted polythiophenes as compared to PPVs involve the enhanced photostability, the broader absorption band, and the ability to self-organize. ${ }^{23-27}$ The organization of polyalkylthiophene backbones has been observed to strongly affect charge transport $^{22,24}$ and may also influence the extent of exciton diffusion. In this work, the photophysical and morphological properties of a combination of the visible-light absorbing electron-accepting porphyrin derivative meso-tetrakis (n-methyl-4-pyridyl) porphyrin tetrachloride ( $\left.\mathrm{H}_{2} \mathrm{TMPyP}\right)$ and regioregular poly(3-hexylthiophene) (P3HT) are presented. The chemical structures of $\mathrm{H}_{2}$ TMPyP and P3HT are shown in Fig. 1(a). The electron-accepting behavior of $\mathrm{H}_{2}$ TMPyP is induced by the presence of quaternized pyridyl side groups, resulting in a reduction potential of $-0.2 \mathrm{eV}$ versus normal hydrogen electrode. ${ }^{28,29}$ Bilayers of $\mathrm{H}_{2}$ TMPyP and electron-donating porphyrins have been studied previously, yielding relatively low incident photon to current efficiencies. $^{29}$ The excellent solubility of P3HT in a solvent in which $\mathrm{H}_{2}$ TMPyP does not dissolve and vice versa allows spincoating of multiple thin films on top of each other. The possibility for solution processing makes this combination of materials of particular interest over bilayers based on, for example, a combination of phthalocyanines and perylene derivatives on $C_{60},{ }^{30-33}$ which need to be formed by evaporation. The absorption spectra of P3HT and $\mathrm{H}_{2}$ TMPyP presented in Fig. 1(b) show that these compounds exhibit complementary absorption bands, which enhances the spectral overlap with the solar emission spectrum.

The surface morphology of the interface between $\mathrm{H}_{2}$ TMPyP and P3HT is studied using atomic force microscopy (AFM). The photovoltaic properties of a photovoltaic cell based on these materials in a bilayer configuration are investigated by current-voltage and impedance measurements. Fitting an analytical model for the IPCE to the experimental data yields $\Lambda_{D}$ in both organic layers. The value for $\Lambda_{D}$ in P3HT differs significantly from values reported in the literature. The difference is attributed to the molecular organization of the P3HT chains, which is characterized by $\mathrm{x}$-ray diffraction. The results show that the molecular organization is a key parameter for the realization of efficient energy transfer in organic photovoltaics.

\section{EXPERIMENT}

\section{A. Sample preparation}

$\mathrm{H}_{2}$ TMPyP and regioregular $\mathrm{P} 3 \mathrm{HT}$ (electronic grade) were purchased from Frontier Scientific (Logan, UT) and Rieke Metals, Inc. (Lincoln, NE), respectively. $\mathrm{H}_{2}$ TMPyP films were prepared in air by spincoating from a solution in $\mathrm{CH}_{3} \mathrm{OH}$ (Aldrich, 99.9\%, $c=10 \mathrm{mg} / \mathrm{ml}$ ) at $2500 \mathrm{rpm}$ onto either a quartz or onto an indium doped $\mathrm{SnO}_{2}$ (ITO) coated glass substrate. Prior to film deposition these substrates were dried at $250{ }^{\circ} \mathrm{C}$ for $1 \mathrm{~h}$. P3HT films were spincoated in air from a solution in $\mathrm{CHCl}_{3}$ at $2500 \mathrm{rpm}$ (anhydrous, 99+\%, Aldrich, $c=15 \mathrm{mg} / \mathrm{ml})$. A Hg contact $\left(A=0.78 \mathrm{~mm}^{2}\right)$ was used as backcontact, ${ }^{34}$ resulting in the device configuration, as presented in the inset of Fig. 2(b). Film thicknesses were determined using a Veeco Dektak 8 Stylus Profiler and equal $25 \pm 5 \mathrm{~nm}$ and $75 \pm 5 \mathrm{~nm}$ for $\mathrm{H}_{2}$ TMPyP and P3HT, respectively.
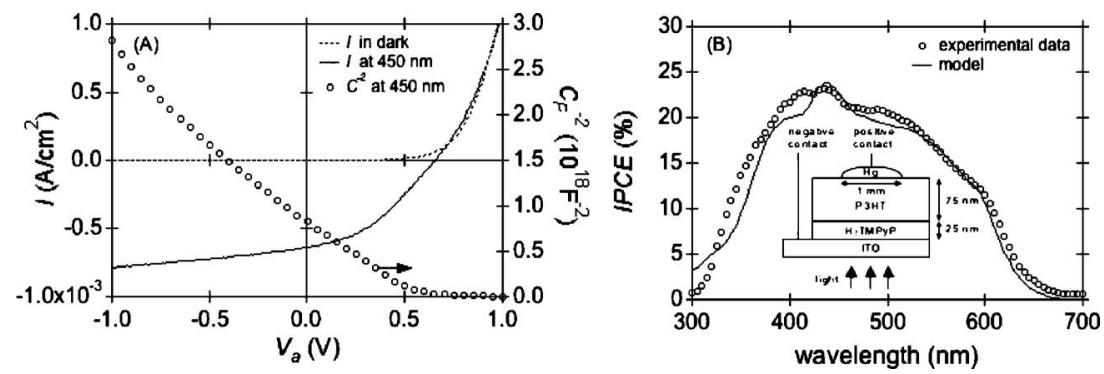

FIG. 2. Current density (dashed is recorded in dark and solid is recorded under illumination with $8 \mathrm{~mW} / \mathrm{cm}^{2}$ at $\left.450 \mathrm{~nm}\right)$ and $C_{P}^{-2}\left(8 \mathrm{~mW} / \mathrm{cm}^{2}\right.$ at $450 \mathrm{~nm}$, recorded at $1 \times 10^{4} \mathrm{~Hz}$ ) of the device presented in the inset of (b) as function of applied voltage (a). The total thickness of the combined layers amounts to $100 \mathrm{~nm}$. (b) shows the IPCE vs wavelength and a fit of Eq. (9) to the experimental data with $\Lambda_{D, \mathrm{H}_{2} \mathrm{TMPyP}}=14 \mathrm{~nm}$ and $\Lambda_{D, \mathrm{P} 3 \mathrm{HT}}=18 \mathrm{~nm}$. 


\section{B. Optical and morphological characterization 1. Optical characterization}

Optical transmission and reflection spectra were recorded on a Perkin Elmer Lambda 900 UV/VIS/NIR spectrometer, using an integrating sphere. The optical absorption coefficient $(\alpha)$ was determined from the transmission and reflection spectra, as described in Ref. 35. Time-resolved fluorescence spectra were recorded with a Lifespec-ps setup using a $405 \mathrm{~nm}$ excitation source (Edinburgh Instruments).

\section{Atomic force microscopy}

Atomic force micrographs were recorded using a Nanoscope atomic force microscope, operated in the tapping mode.

\section{X-ray diffraction analysis}

$\mathrm{X}$-ray diffraction measurements were performed in the Bragg-Brentano mode on a Bruker D8 advance x-ray diffractometer, equipped with a $\mathrm{Cu}$ anode $\left(\lambda_{K \alpha}=1.5408 \AA\right)$ operated at $40 \mathrm{kV}$ and $30 \mathrm{~mA}$.

\section{Photovoltaic and Mott-Schottky characterization}

Photocurrents were recorded in air, using an Autolab PGSTAT 10 EcoChemie potentiostat controlled by GPES 3 software in combination with a Xe lamp (Eurosep Instruments) equipped with a monochromator (Triax series 0144B 99/236). The incident power $\left(P_{\text {in }}\right)$ varied from 6 to $8 \mathrm{~mW} / \mathrm{cm}^{2}$ for the wavelength range of $300-700 \mathrm{~nm}$. Shortcircuit currents were measured using a Keithley 2700 Multimeter. The fill factor (FF) was determined using

$$
\mathrm{FF}=\frac{V_{\max } I_{\max }}{V_{\mathrm{OC}} I_{\mathrm{SC}}},
$$

where $V_{\max }$ and $I_{\max }$ represent the voltage and the current at the maximum power point, $V_{\mathrm{OC}}$ is the open-circuit voltage, and $I_{\mathrm{SC}}$ is the short-circuit voltage. The incident photon to current efficiency (IPCE) as function of wavelength is determined using

$$
\mathrm{IPCE}=\frac{I_{\mathrm{SC}} h c}{P_{\text {in }} e \lambda} 100 \%,
$$

where $P_{\text {in }}$ represents the power of the incident light. ${ }^{36}$

\section{RESULTS AND DISCUSSION}

\section{A. Photovoltaic properties}

Figure 2(a) shows the current-voltage characteristics of the photovoltaic device based on $\mathrm{H}_{2}$ TMPyP and P3HT in dark and under monochromatic illumination at $450 \mathrm{~nm}$ with an incident power $\left(P_{\text {in }}\right)$ of $8 \mathrm{~mW} / \mathrm{cm}^{2}$. The configuration of the photovoltaic device is shown in the inset of Fig. 2(b). The forward bias direction corresponds to a negative voltage on the ITO electrode. Under this illumination the opencircuit voltage $\left(V_{\mathrm{OC}}\right)$ equals $0.65 \mathrm{~V}$, the short-circuit current $\left(I_{\mathrm{SC}}\right)$ is $0.64 \mathrm{~mA} / \mathrm{cm}^{2}$, and the fill factor $(\mathrm{FF})$ calculated from Eq. (3) is low and amounts to 0.36. Figure 2(b) shows the wavelength dependence of the IPCE determined using
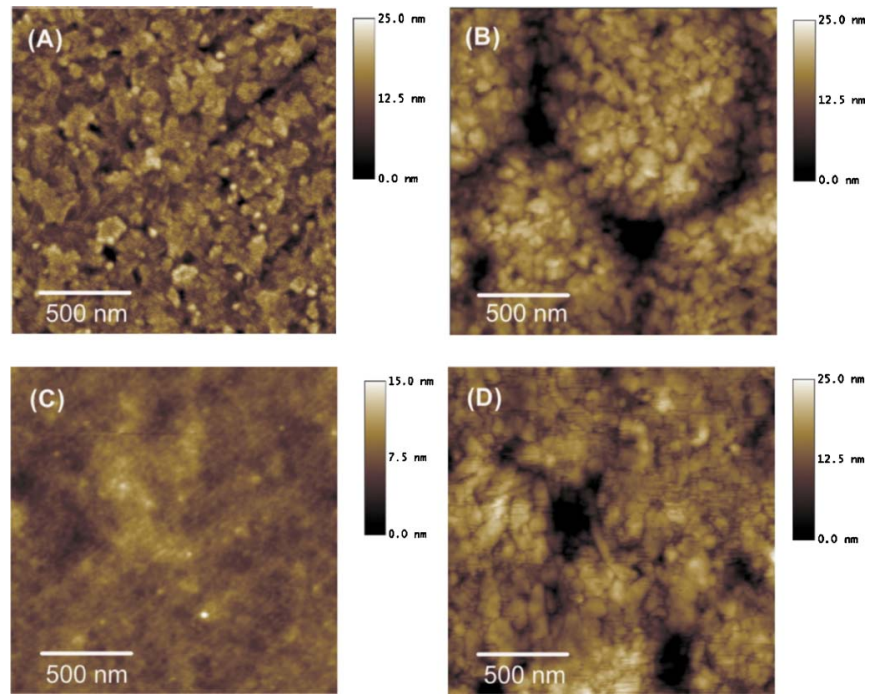

FIG. 3. (Color online) Atomic force micrographs of ITO (a), the sample as shown in (a) spincoated with $\mathrm{H}_{2} \mathrm{TMPyP}$ (b), the sample as shown in (b) spincoated with P3HT (c), and the sample as shown in (c) after removing the P3HT layer with $\mathrm{CHCl}_{3}$ (d). Note the smaller scale used for (c).

Eq. (4). The IPCE value exceeds $20 \%$ over a broad wavelength regime. Comparison of the IPCE spectrum with the absorption spectra of $\mathrm{H}_{2}$ TMPyP and P3HT presented in Fig. 1(b) clearly shows that both materials contribute to the photocurrent. This implies that excitons formed in $\mathrm{H}_{2}$ TMPyP and in P3HT are dissociated into charge carriers. The contribution originating from P3HT to the IPCE considerably exceeds values reported earlier for smooth bilayer devices based on polythiophene derivatives and $C_{60}{ }^{37,38}$ Possible explanations for the unexpected high IPCE values observed for the photovoltaic cell based on $\mathrm{H}_{2}$ TMPyP and P3HT are discussed in Secs. III E and III F.

\section{B. Interface morphologies}

The average distance an exciton has to travel before it can be dissociated into charge carriers is determined by the smoothness of the interface between $\mathrm{H}_{2}$ TMPyP and P3HT. Figures 3(a)-3(d) show the surface morphology of the ITO coated glass, the $\mathrm{H}_{2}$ TMPyP spincoated onto the ITO electrode, the sample shown in (b) spincoated with a P3HT layer, and the sample presented in (c) after dissolving the P3HT layer specifically using $\mathrm{CHCl}_{3}$, respectively. The surface morphology of the original $\mathrm{H}_{2}$ TMPyP layer appears to be almost identical to the surface morphology of $\mathrm{H}_{2}$ TMPyP after the deposition of P3HT and the removal of the P3HT layer. This demonstrates the absence of any significant changes in surface morphology of $\mathrm{H}_{2}$ TMPyP upon the deposition of the P3HT layer. Spincoating of P3HT onto $\mathrm{H}_{2}$ TMPyP therefore yields a bilayer configuration, as presented schematically in the inset of Fig. 2(b), rather than an interpenetrating network of both materials. As a consequence, the high IPCE values observed in this work demonstrate the presence of efficient energy transfer pathways toward the exciton dissociating interface. 


\section{Impedance measurements}

Apart from efficient exciton diffusion through the $\mathrm{H}_{2}$ TMPyP and P3HT layers and exciton dissociation at the interface, the high IPCE values also indicate efficient collection of charge carriers at the electrodes. Transport of charge carriers from the photoactive interface through the organic layers toward the electrodes can occur by drift induced by the internal electric field and/or by diffusion. The presence and width of a depletion layer near the porphyrin/polymer interface are investigated by using the impedance measurements. The complex impedance spectra of the bilayer device at various applied voltages are presented in Appendix A. Current/voltage plots recorded on the individual organic layers do not show a diodelike behavior. In addition, no voltage dependence capacitance is observed for the individual organic layers, indicating the presence of near Ohmic contacts. In contrast, the presence of a diodelike behavior and a voltage dependent capacitance in the case of the bilayer indicates the formation of a junction at the interface of the two organic layers. A simple equivalent circuit consisting of a capacitor with capacitance $C_{P}$ parallel to a resistor with resistance $R_{P}$ in series with a resistor $\left(R_{S}\right)$ is sufficient to fit the impedance spectra. The fits and fit parameters are included in Appendix A. The capacitance appears to be a function of the applied voltage $\left(V_{a}\right)$. The width of the depletion layer $(w)$ can be determined from the capacitance by using

$$
w=\frac{\varepsilon_{r} \varepsilon_{0} A}{C_{p}},
$$

where $A$ represents the electrode area, $\varepsilon_{r}$ the relative dielectric constant, and $\varepsilon_{0}$ the permittivity of free space. The relative dielectric constants of $\mathrm{P} 3 \mathrm{HT}$ and $\mathrm{H}_{2}$ TMPyP are assumed to be similar and taken equal to a value of $3 .^{29,39}$ From the observed capacitance of $1 \mathrm{nF}$ at $V_{a}=0 \mathrm{~V}$ and $A$ $=0.78 \mathrm{~mm}^{2}$ follows a total depletion width of $20 \mathrm{~nm}$. The dependence of the capacitance of the depletion layer on the applied voltage is described by ${ }^{40}$

$$
\frac{1}{C_{P}^{2}}=\frac{2}{e \varepsilon_{r} \varepsilon_{0} A^{2}}\left(\frac{1}{N_{\mathrm{P} 3 \mathrm{HT}}}+\frac{1}{N_{\mathrm{H}_{2} \mathrm{TMPyP}}}\right)\left(V_{\mathrm{BI}}-V_{a}\right),
$$

where $e$ represents the electronic charge, $N_{\mathrm{P} 3 \mathrm{HT}}$ the concentration of ionized impurities in the P3HT layer, $N_{\mathrm{H}_{2} \text { TMPyP }}$ the concentration of ionized impurities in the $\mathrm{H}_{2}$ TMPyP layer, and $V_{\mathrm{BI}}$ the built-in potential present over the depletion layer. A Mott-Schottky plot $\left(C_{P}^{-2}\right.$ vs $\left.V_{a}\right)$ recorded at $10 \mathrm{kHz}$ under monochromatic illumination at $450 \mathrm{~nm}$ with an incident power of $8 \mathrm{~mW} / \mathrm{cm}^{2}$ is included in Fig. 2(a). From Eq. (6) and the experimental Mott-Schottky data follows a built-in potential of $0.55 \mathrm{~V}$, close to the $V_{\mathrm{OC}}$ observed. In case $N_{\text {P3HT }}=N_{\mathrm{H}_{2} \text { TMPyP }}$, the depletion layer is located to equal extents over both layers. ${ }^{40}$ From Fig. 2(a), it is clear that at $V_{a}=-1.0 \mathrm{~V}$ the bilayer device is not yet fully depleted. At this potential the observed capacitance is close to $0.6 \mathrm{nF}$, which corresponds to a $w$ of $35 \mathrm{~nm}$. This value exceeds the thickness of the $\mathrm{H}_{2}$ TMPyP layer. From this observation it is inferred that the depletion region is not only located in the $\mathrm{H}_{2}$ TMPyP layer but also in the P3HT layer. This indicates that the concentration of ionized impurities in the P3HT

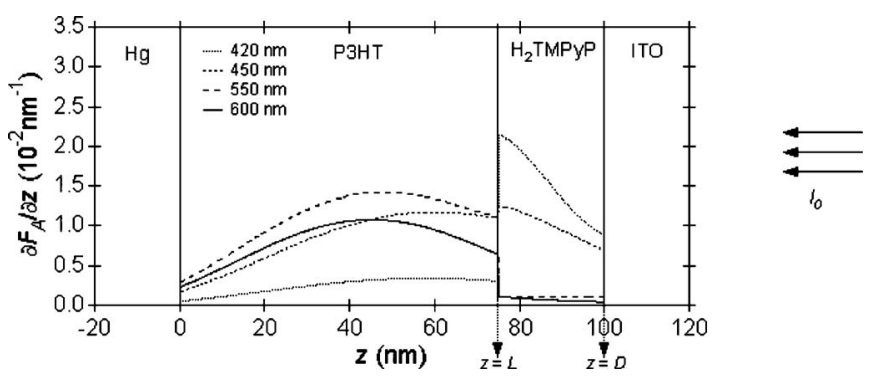

FIG. 4. First derivative of $F_{A}$ with respect to $z$ within the bilayer device presented in the inset in Fig. 2(b) at selected wavelengths, determined using the optical modeling program scouT.

layer is either in the same order of magnitude or is lower than the concentration in the $\mathrm{H}_{2} \mathrm{TMPyP}$ layer. The presence of mobile holes in P3HT layers is generally attributed to reversible doping with oxygen, ${ }^{41-44}$ while the origin of the excess mobile electrons in the $\mathrm{H}_{2}$ TMPyP layer is not clearly established. ${ }^{45}$ The internal electric field present over the interface between $\mathrm{H}_{2}$ TMPyP and P3HT is expected to retard interfacial recombination of electrons and holes formed on exciton dissociation.

\section{Determination of the exciton root-mean-square displacement}

Prime parameters that determine the IPCE of a bilayer device are the value of $\Lambda_{D}$ and the fraction of absorbed light $\left(F_{A}\right)$ in the individual layers. The absorption profile within the bilayer device is determined using the optical modeling program SCOUT, ${ }^{46}$ assuming the interface between $\mathrm{H}_{2}$ TMPyP and P3HT to be perfectly smooth. More details about the scouT modeling are given in Appendix B. Figure 4 shows the first derivative of $F_{A}$ with respect to the distance from the mercury contact $(z)$ for selected wavelengths. Especially within the $\mathrm{H}_{2}$ TMPyP layer, the absorption profile turns out to deviate considerably from a Lambert-Beer profile, which mainly originates from the presence of the metal contact. The number of excitons formed that are able to reach the photoactive interface can be deduced from solving the following differential equation for exciton diffusion: ${ }^{47}$

$$
\frac{\partial n_{i}(z)}{\partial t}=I_{0} \frac{\partial F_{A, i}(z)}{\partial z}-\frac{n_{i}(z)}{\tau_{E, i}}+D_{E, i} \frac{\partial^{2} n_{i}(z)}{\partial z^{2}},
$$

where $n_{i}(z)$ is the exciton concentration at a depth $z$ within layer $i$ and $I_{0}$ is the incident light intensity. Since a continuous light source is used, the first derivative of $n_{i}(z)$ with respect to $t$ is zero. In addition, excitons that reach the interface with an electrode are assumed to be rapidly quenched and those that reach the interface between $\mathrm{H}_{2}$ TMPyP and P3HT to be rapidly dissociated into charge carriers, i.e., $n_{i}$ $=0$ at these interfaces. Solving Eq. (7) under these conditions and using the absorption profiles determined by SCOUT yield $n_{i}(z)$. The fraction of $I_{0}$ that leads to excitons that reach the photoactive interface $\left(S_{i}\right)$ can be expressed by 


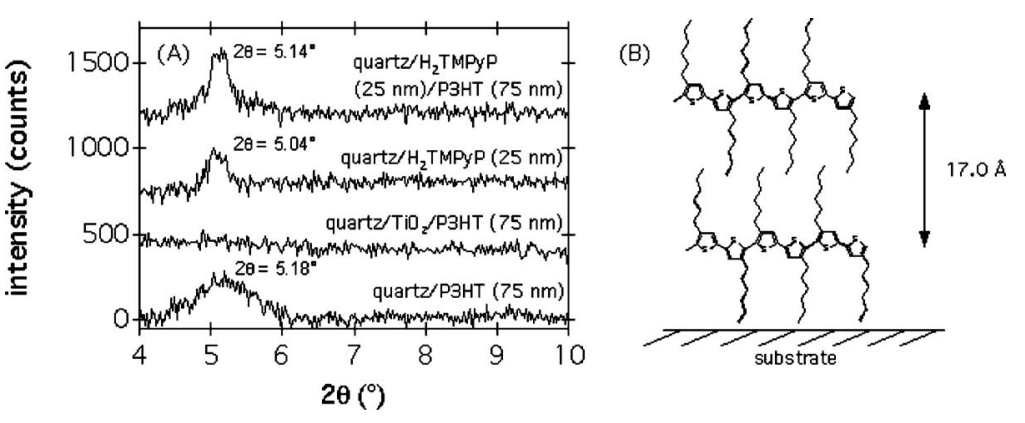

FIG. 5. X-ray diffraction patterns of quartz/P3HT (75 nm), quartz/ $\mathrm{H}_{2}$ TMPyP (25 nm), quartz/ $\mathrm{H}_{2}$ TMPyP (25 $\mathrm{nm}) / \mathrm{P} 3 \mathrm{HT}(75 \mathrm{~nm})$, and quartz/ $\mathrm{TiO}_{2} / \mathrm{P} 3 \mathrm{HT}(75 \mathrm{~nm})$ (a). The X-ray diffraction intensity observed for a bare $\mathrm{TiO}_{2}$ / quartz substrate is subtracted from the latter pattern, and the other patterns are corrected for the diffraction intensity of a bare quartz substrate. Note that the data presented have different offsets to improve clarity. (b) shows the alignment of P3HT backbones parallel with the hexyl side chains perpendicular to a substrate.

$$
S_{i}=-\left.D_{E, i} \frac{\partial n_{i}(z)}{\partial z} \frac{1}{I_{0}}\right|_{z=L},
$$

where $L$ is the $z$ coordinate corresponding to the interface between $\mathrm{H}_{2}$ TMPyP and P3HT. More details about the solution of Eqs. (7) and (8) are presented in Appendix C. The total number of excitons that reaches the photoactive interface is the sum of the number of excitons in each layer that reaches this interface. Hence, the total amount of excitons reaching the interface divided by $I_{0}$ is defined as the sum of the $S_{i}$ factors of each layer. For expressing the IPCE in terms of $S_{i}$, the charge separation yield at the interface into free charge carriers is assumed to be unity. Furthermore, it is assumed that all charge carriers formed on exciton dissociation are collected by the electrodes. Under these conditions, the IPCE of the bilayer device follows from

$$
\mathrm{IPCE}=\left(S_{\mathrm{P} 3 \mathrm{HT}}+S_{\mathrm{H}_{2} \text { TMPyP }}\right) 100 \% .
$$

Fitting Eq. (9) to the experimental IPCE spectrum, with $\tau_{E}$ 290 ps for P3HT and 1.28 ns for $\mathrm{H}_{2}$ TMPyP as obtained from the fluorescence decay measurements (data not shown), results in the fit are included in Fig. 2(b) with $D_{E, \mathrm{H}_{2} \mathrm{TMPyP}}=8$ $\times 10^{-8} \mathrm{~m}^{2} / \mathrm{s}$ and $D_{E, \mathrm{P} 3 \mathrm{HT}}=5.9 \times 10^{-7} \mathrm{~m}^{2} / \mathrm{s}$. Combining these values for $D_{E}$ with the observed exciton lifetimes and using Eq. (1) yields $\Lambda_{D, \mathrm{H}_{2} \mathrm{TMPyP}}=14 \mathrm{~nm}$ and $\Lambda_{D, \mathrm{P} 3 \mathrm{HT}}$ $=18 \mathrm{~nm}$. This value for $\Lambda_{D \text {, Р3HT }}$ is significantly larger than previous values for polythiophene derivatives deposited onto $C_{60}$ (Refs. 37 and 48) or $\mathrm{TiO}_{2}{ }^{8}{ }^{8}$ where values close to $7 \mathrm{~nm}$ (Ref. 12) are reported. Possible reasons for the discrepancy in $\Lambda_{D}$ between those systems and the bilayer investigated in this study are discussed below.

\section{E. Determination of the molecular organization}

The discrepancy in $\Lambda_{D \text {, Р3HT }}$ for P3HT deposited onto $\mathrm{H}_{2}$ TMPyP and the previous reported values most likely originate from differences in the molecular organization. The degree of molecular organization in a thin film is elucidated using $\mathrm{x}$-ray diffraction. Figure 5(a) shows the X-ray diffraction patterns of single layers of $\mathrm{H}_{2}$ TMPyP and P3HT deposited on quartz and a bilayer of $\mathrm{H}_{2}$ TMPyP and P3HT on quartz. For reference purposes, the pattern observed for P3HT deposited onto a similar $\mathrm{TiO}_{2}$ / quartz substrate as used in Ref. 8 is also illustrated, for which $\Lambda_{D \text {,Р } 3 \mathrm{HT}}$ has been determined to be $7 \mathrm{~nm}^{8}$ The $\mathrm{x}$-ray diffraction intensity observed for a bare $\mathrm{TiO}_{2}$ / quartz substrate is subtracted from the latter pattern, and the other patterns are corrected for the diffraction intensity of a bare quartz substrate. The x-ray dif- fraction pattern of a single layer of P3HT on quartz shows a diffraction peak at $2 \theta=5.18^{\circ}$. In the case of first order diffraction, this peak reveals the presence of a well-developed structure directed perpendicular to the quartz substrate with an interplanar distance equal to $17.0 \AA$. This distance most likely corresponds to the spacing between P3HT chains along the hexyl side chains, as reported in several earlier works $^{23,25-27}$ and shown schematically in Fig. 5(b). In contrast to a P3HT layer on quartz, for P3HT deposited on $\mathrm{TiO}_{2} /$ quartz, hardly any diffraction peak is observed, indicating a relatively low degree of organization of the P3HT chains for the latter system. The difference in degree of molecular organization could either originate from variations in the roughness of the substrate surface or from the interaction of P3HT with the surface functionalities of the substrate. From AFM analysis follows only a slightly higher surface roughness of $\mathrm{TiO}_{2}$ as compared to quartz (see Appendix D). This leads to the conclusion that the difference in degree of organization of $\mathrm{P} 3 \mathrm{HT}$ chains on $\mathrm{TiO}_{2}$ and on quartz is most likely due to the interaction of the conjugated polymer with surface functionalities of the substrate.

Remarkably, a layer of $\mathrm{H}_{2}$ TMPyP deposited on quartz possesses a diffraction peak at almost a similar angle as observed for P3HT on quartz. The maximum diffraction is observed at $2 \theta=5.04^{\circ}$, corresponding to the case of first order diffraction to an interplanar distance equal to $17.5 \AA$. Since this distance equals the diameter of a $\mathrm{H}_{2}$ TMPyP molecule, the observed peak suggests the alignment of the $\mathrm{H}_{2}$ TMPyP molecular planes perpendicular to the substrate. As compared to a bare $\mathrm{H}_{2}$ TMPyP layer, the bilayer of $\mathrm{H}_{2}$ TMPyP and P3HT deposited on quartz possesses a more intense diffraction peak, which is attributed to a contribution from both organic layers. The degree of molecular order of P3HT chains deposited on $\mathrm{H}_{2}$ TMPyP is hence higher than for $\mathrm{P} 3 \mathrm{HT}$ on $\mathrm{TiO}_{2}$, even though the surface of the latter substrate is smoother (see Appendix D). It should be noted that quartz and $\mathrm{H}_{2}$ TMPyP possess a stronger hydrophilicity as compared to $\mathrm{TiO}_{2},{ }^{49-51}$ which may explain the higher degree of order of P3HT deposited onto the first two materials. The enhanced degree of molecular order of P3HT chains deposited onto $\mathrm{H}_{2}$ TMPyP as compared to $\mathrm{TiO}_{2}$ can explain the long exciton root-mean-square displacement observed for the first system.

\section{F. Origin of the long exciton root-mean-square displacement}

The value for $\Lambda_{D}$ depends on the exciton diffusion coefficient $\left(D_{E}\right)$ and the exciton lifetime $\left(\tau_{E}\right)$ according to Eq. 
TABLE I. Parameters characterizing the energy transfer in $\mathrm{H}_{2}$ TMPyP, P3HT deposited on $\mathrm{H}_{2}$ TMPyP, and P3HT deposited on $\mathrm{TiO}_{2}$.

\begin{tabular}{cccccc}
\hline \hline \multicolumn{1}{c}{ Layer } & $\Lambda_{D}(\mathrm{~nm})$ & $\tau_{E}(s)$ & $D_{E}\left(\mathrm{~m}^{2} / \mathrm{s}\right)$ & $R_{\mathrm{DA}}(\AA)$ & $k_{\mathrm{ET}}\left(s^{-1}\right)$ \\
\hline $\mathrm{H}_{2}$ TMPyP & 14 & $1.28 \times 10^{-9}$ & $8 \times 10^{-8}$ & 17.5 & $3 \times 10^{10}$ \\
P3HT on $\mathrm{H}_{2}$ TMPyP & 18 & $2.90 \times 10^{-10}$ & $5.9 \times 10^{-7}$ & 17.0 & $2 \times 10^{11}$ \\
P3HT on $\mathrm{TiO}_{2}$ & $7^{\mathrm{a}}$ & $2.90 \times 10^{-10}$ & $8 \times 10^{-8}$ & 17.0 & $3 \times 10^{10}$ \\
\hline \hline
\end{tabular}

${ }^{\mathrm{a}}$ From Ref. 8

(1). In case an exciton is localized on a single molecule, $D_{E}$ depends on the energy transfer rate $\left(k_{\mathrm{ET}}\right)$ and the center-tocenter distance between adjacent molecules $\left(R_{\mathrm{DA}}\right)$ according to

$$
D_{E}=k_{\mathrm{ET}} R_{\mathrm{DA}}^{2},
$$

The exciton lifetimes are determined from fluorescence decay measurements (data not shown). The exciton diffusion coefficients in the individual layers are deduced from the experimental IPCE values, as described in Sec. III D. Combining the exciton diffusion coefficient with the interplanar distance determined by x-ray diffraction and using Eq. (10) yield the energy transfer rate. All these parameters characterizing the energy transfer in $\mathrm{H}_{2}$ TMPyP, P3HT deposited on $\mathrm{H}_{2} \mathrm{TMPyP}$, and $\mathrm{P} 3 \mathrm{HT}$ deposited on $\mathrm{TiO}_{2}$ are presented in Table I. Note that the energy transfer rate between the disordered P3HT chains on $\mathrm{TiO}_{2}$ is a factor of 7 lower than the value determined for organized P3HT chains deposited onto $\mathrm{H}_{2}$ TMPyP.

In the limit of weak intermolecular excitonic coupling, motion of excitons can be considered as diffusive with an intermolecular hopping rate $k_{\mathrm{ET}}$ given by ${ }^{52,53}$

$$
k_{\mathrm{ET}}=\frac{2 \pi}{h}\left|V_{\mathrm{DA}}\right|^{2} J_{\mathrm{DA}}
$$

where the factor $V_{\mathrm{DA}}$ denotes the excitonic coupling between the energy donor and the energy acceptor, and $J_{\mathrm{DA}}$ represents the spectral overlap integral. The definition and evaluation of the spectral overlap integrals for $\mathrm{H}_{2}$ TMPyP and P3HT are specified in Appendix E. The experimental absorption and fluorescence spectra yield values for $J_{\mathrm{DA}}$ equal to 4.3 $\times 10^{18} \mathrm{~J}^{-1}$ for $\mathrm{H}_{2}$ TMPyP and $5.5 \times 10^{17} \mathrm{~J}^{-1}$ for P3HT. Combining this with the observed values for $k_{\mathrm{ET}}$ yields values for the excitonic coupling $V_{\mathrm{DA}}$ of $16 \mathrm{~cm}^{-1}$ for $\mathrm{H}_{2}$ TMPyP, $125 \mathrm{~cm}^{-1}$ for P3HT deposited on $\mathrm{H}_{2}$ TMPyP, and $47 \mathrm{~cm}^{-1}$ for $\mathrm{P} 3 \mathrm{HT}$ on $\mathrm{TiO}_{2}$. The stronger excitonic coupling observed for $\mathrm{P} 3 \mathrm{HT}$ on $\mathrm{H}_{2} \mathrm{TMPyP}$ as compared to $\mathrm{P} 3 \mathrm{HT}$ on $\mathrm{TiO}_{2}$ is most likely due to a higher and more favorable degree of molecular organization of the $\mathrm{P} 3 \mathrm{HT}$ chains found for the first system. The value of $V_{\mathrm{DA}}$ equal to $125 \mathrm{~cm}^{-1}$ found for P3HT deposited on $\mathrm{H}_{2}$ TMPyP indicates a strong excitonic coupling between the adjacent $\mathrm{P} 3 \mathrm{HT}$ chains that are organized, as shown in Fig. 5(b). Quantum-chemical calculations on excitonic couplings are underway in order to establish to which extent the value for the excitonic coupling can be understood on the basis of this molecular organization and whether exciton transfer can be further improved.

\section{CONCLUSIONS}

This work presents the morphological and photophysical properties of a bilayer of the electron-accepting porphyrin derivative $\mathrm{H}_{2}$ TMPyP and P3HT. A photovoltaic cell based on such a bilayer exhibits an IPCE over $20 \%$ over a broad wavelength regime. Fitting a model for one-dimensional exciton diffusion in the direction perpendicular to the interface between $\mathrm{H}_{2}$ TMPyP and P3HT, which includes the absorption profiles in the bilayer to the experimental IPCE values, gives the exciton root-mean-square displacement $\left(\Lambda_{D}\right)$ in each layer. From x-ray diffraction, it is inferred that the $\mathrm{H}_{2}$ TMPyP molecular cores are aligned perpendicular to the interface with P3HT, while the P3HT backbones are aligned parallel to the interface. For the $\mathrm{H}_{2}$ TMPyP layer $\Lambda_{D}$ amounts to $14 \mathrm{~nm}$, while a value of $18 \mathrm{~nm}$ is found for the P3HT layer. Combining the $\Lambda_{D}$ values with the intermolecular distances and the exciton lifetimes observed yields the intermolecular energy transfer rates, which amount to $3 \times 10^{10} \mathrm{~s}^{-1}$ for $\mathrm{H}_{2}$ TMPyP and $2 \times 10^{11} \mathrm{~s}^{-1}$ for P3HT. From application of Fermi's golden rule to these energy transfer rates follows an excitonic coupling between $\mathrm{H}_{2}$ TMPyP molecules that amounts to $16 \mathrm{~cm}^{-1}$, while for P3HT a value as high as $125 \mathrm{~cm}^{-1}$ is found. The latter value is almost a factor of 3 higher than the excitonic coupling between the disordered P3HT chains determined to be $47 \mathrm{~cm}^{-1}$. This illustrates the importance of controlling the molecular organization for the realization of efficient energy transfer in organic optoelectronics.

\section{ACKNOWLEDGMENTS}

Dr. Albert Goossens is acknowledged for helpful discussions. Murat Durkut and Menno Poot from the Molecular Electronics and Devices group, Kavli Institute of Nanoscience, TU Delft are acknowledged for assistance with the AFM measurements. The research was supported financially by the Delft Research Centre for Sustainable Energy. A.S. gratefully acknowledges Senter Novem for financial support.

\section{APPENDIX A}

Figure 6 shows the impedance spectra of the device shown in the inset of Fig. 2(b) recorded at various applied voltages and fits of an equivalent circuit of a capacitor $C_{P}$ parallel to a resistor $R_{P}$ in series with a resistor $R_{S}$.

\section{APPENDIX B}

The real and complex parts $(n)$ and $(k)$ of the refractive index of the materials used are determined from the experimental transmission and reflection spectra using the program 

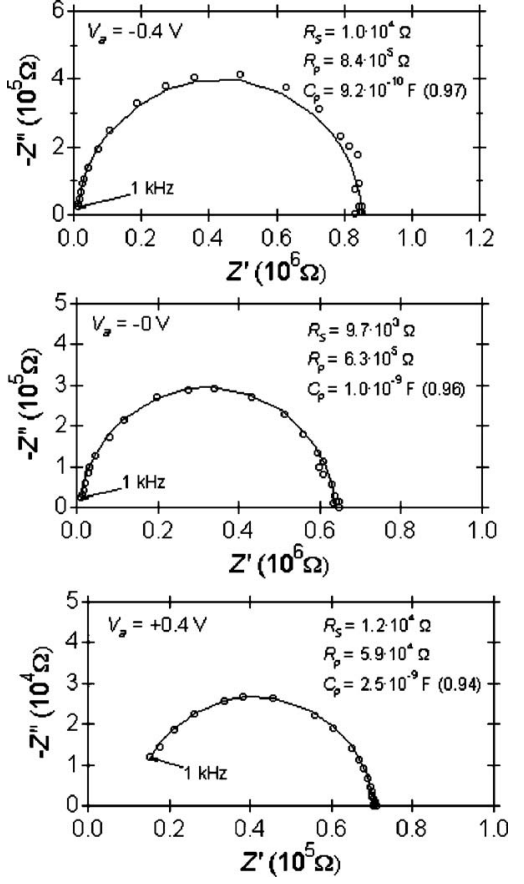

FIG. 6. Impedance spectra of the device presented in the inset of Fig. 2(b) $\left(8 \mathrm{~mW} / \mathrm{cm}^{2}\right.$ at $\left.450 \mathrm{~nm}\right)$ recorded at $V_{a}=-0.4 \mathrm{~V}, V_{a}=0 \mathrm{~V}$, and $V_{a}=$ $+0.4 \mathrm{~V}$ and fits of an equivalent circuit of a capacitor $C_{P}$ parallel to a resistor $R_{P}$ in series with a resistor $R_{S}$ with parameters shown in the insets. The nonidealities of the capacitors are given between brackets.

SCOUT. ${ }^{46}$ Figure 7(a) shows the values for $n$ and $k$ as function of wavelength for quartz, $\mathrm{H}_{2}$ TMPyP, and P3HT. The fractions of transmitted light $\left(F_{T}\right)$ and reflected light $\left(F_{R}\right)$ of quartz/ $\mathrm{H}_{2}$ TMPyP $(25 \mathrm{~nm}) / \mathrm{P} 3 \mathrm{HT}(75 \mathrm{~nm})$, illuminated from the side of the P3HT film, are determined using SCOUT on basis of $n$ and $k$ of the individual materials. The modeled $F_{T}$ and $F_{R}$ are shown in Fig. 7(b) and agree very well with the experimental data. This validates modeling the absorption profile within the device presented in the inset of Fig. 2(b) using SCOUT. The values for $n$ and $k$ of ITO coated glass are determined from the experimental transmission and reflection spectra and $n$ and $k$ of $\mathrm{Hg}$ are taken from Ref. 54 and shown in Fig. 7(c).

\section{APPENDIX C}

The first derivative of $F_{A}$ with respect to $z$ determined using SCOUT has been converted to a sinusoidal equation, leading to the following steady-state differential equation for exciton diffusion:
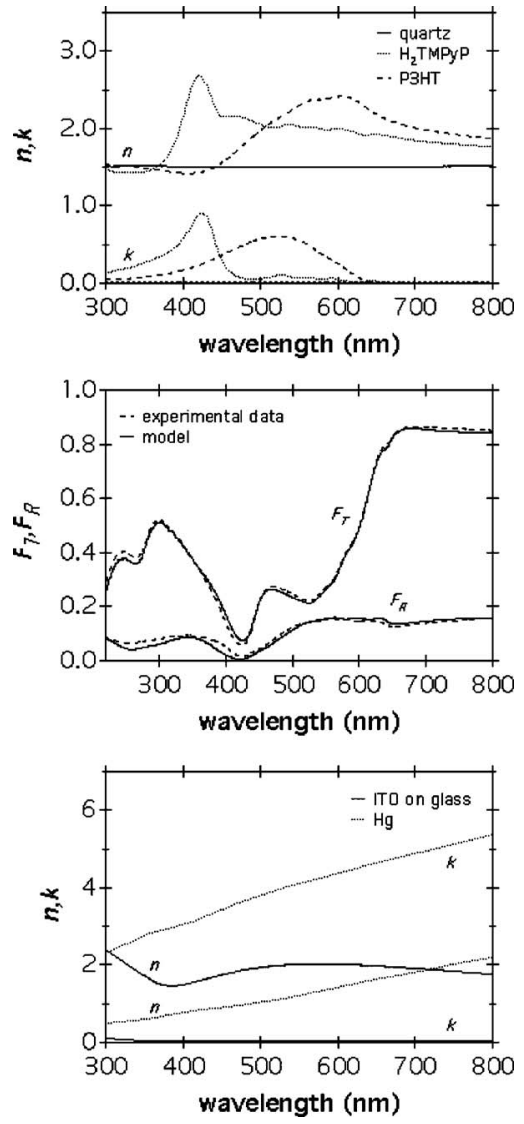

FIG. 7. Optical constants of quartz, $\mathrm{H}_{2} \mathrm{TMPyP}$, and $\mathrm{P} 3 \mathrm{HT}$ (top), experimental and modeled fraction of transmitted $\left(F_{T}\right)$ and reflected light $\left(F_{R}\right)$ of quartz/ $\mathrm{H}_{2}$ TMPyP $(25 \mathrm{~nm}) / \mathrm{P} 3 \mathrm{HT}(75 \mathrm{~nm})$ illuminated from the side of the P3HT film (middle) and optical constants of ITO on glass and Hg (from Ref. 54) (bottom).

$$
I_{0}\left[A_{i} \sin \left(f_{i} z+\phi_{i}\right)\right]-\frac{n_{i}(z)}{\tau_{E, i}}+D_{E, i} \frac{\partial^{2} n_{i}(z)}{\partial z^{2}}=0 .
$$

The boundary conditions at the interface between an organic layer and an electrode and at the interface between $\mathrm{H}_{2}$ TMPyP and P3HT have to be defined to solve Eq. (C1). Excitons reaching the interface with an electrode are assumed to be rapidly quenched and those that reach the photoactive interface to be rapidly dissociated into charge carriers. For the P3HT layer this implies that the concentration of excitons is zero at $z=0$ and $z=L$ (see Fig. 4). Solving Eq. (C1) under these conditions by using the program MAPLE results in

$$
\begin{aligned}
& n_{i}(z)=\frac{\left(\sin \left(f_{i} L+\phi\right) e^{(z+L) / \sqrt{D_{E, i} \tau_{E, i}}}-\sin (\phi) e^{z / \sqrt{D_{E, i} \tau_{E, i}}}+\sin (\phi) e^{\left.-(z-2 L) / \sqrt{D_{E, i} \tau_{E, i}}\right)} I_{0} A_{i} \tau_{E, i}\right.}{\left(1+f_{i}^{2} D_{E, i} \tau_{E, i}\right)\left(1-e^{\left.2 L / \sqrt{D_{E, i} \tau_{E, i}}\right)}\right.} \\
& +\frac{\left(-\sin \left(f_{i} L+\phi\right) e^{-(z-L) / \sqrt{D_{E, i} \tau_{E, i}}}+\sin \left(f_{i} z+\phi\right)-\sin \left(f_{i} z+\phi\right) e^{\left.2 L / \sqrt{D_{E, i} \tau_{E, i}}\right) I_{0} A_{i} \tau_{E, i}},\right.}{\left(1+f_{i}^{2} D_{E, i} \tau_{E, i}\right)\left(1-e^{2 L / \sqrt{D_{E, i} \tau_{E, i}}}\right)},
\end{aligned}
$$

with $i=\mathrm{P} 3 \mathrm{HT}$. Analogously, for the $\mathrm{H}_{2}$ TMPyP layer the concentration of excitons is equal to zero at $z=L$ and $z=D$, where $D$ is the $z$ coordinate corresponding to the interface between $\mathrm{H}_{2}$ TMPyP and ITO, leading to 


$$
\begin{aligned}
n_{i}(z)= & \frac{\left(\sin \left(f_{i} L+\phi\right) e^{(z+L) / \sqrt{D_{E, i} \tau_{E, i}}}-\sin \left(f_{i} D+\phi\right) e^{(z+D) / \sqrt{D_{E, i} \tau_{E, i}}}+\sin \left(f_{i} D+\phi\right) e^{\left.-(z-D-2 L) / \sqrt{D_{E, i} \tau_{E, i}}\right) I_{0}} A_{i} \tau_{E, i}\right.}{\left(1+f_{i}^{2} D_{E, i} \tau_{E, i}\right)\left(e^{2 D / \sqrt{D_{E, i} \tau_{E, i}}}-e^{\left.2 L / \sqrt{D_{E, i} \tau_{E, i}}\right)}\right.} \\
& +\frac{\left(-\sin \left(f_{i} L+\phi\right) e^{-(z-2 D-L) / \sqrt{D_{E, i} \tau_{E, i}}}+\sin \left(f_{i} z+\phi\right) e^{2 D / \sqrt{D_{E, i} \tau_{E, i}}}-\sin \left(f_{i} z+\phi\right) e^{\left.2 L / \sqrt{D_{E, i} \tau_{E, i}}\right) I_{0} A_{i} \tau_{E, i}}\right.}{\left(1+f_{i}^{2} D_{E, i} \tau_{E, i}\right)\left(e^{2 D / \sqrt{D_{E, i} \tau_{E, i}}}-e^{2 L / \sqrt{D_{E, i} \tau_{E, i}}}\right)},
\end{aligned}
$$

with $i=\mathrm{H}_{2}$ TMPyP. The fraction of incident photons that leads to excitons reaching the photoactive interface by diffusion $\left(S_{i}\right)$ is expressed by

$$
S_{i}=-\left.D_{E, i} \frac{\partial n_{i}(z)}{\partial z} \frac{1}{I_{0}}\right|_{z=L}
$$

Application of Eq. (C4) to Eqs. (C2) and (C3) results in the following analytical expressions for the device, with $i=\mathrm{P} 3 \mathrm{HT}$ (C5) and $i=\mathrm{H}_{2}$ TMPyP (C6), respectively:

$$
\begin{aligned}
& S_{i}=\frac{\left(D_{E, i} \cos \left(f_{i} L+\phi\right) f_{i} \tau_{i} e^{2 L / \sqrt{D_{E, i} \tau_{E, i}}}-D_{E, i} \cos \left(f_{i} L+\phi\right) f_{i} \tau_{i}\right)}{\left(\frac{1+f_{i}^{2} \tau_{i} D_{E, i}}{A_{i}}\right)\left(-e^{2 L / \sqrt{D_{E, i} \tau_{E, i}}}+1\right)} \\
& +\frac{\left(2 \sqrt{D_{E, i} \tau_{E, i}} \sin (\phi) e^{L / \sqrt{D_{E, i} \tau_{E, i}}}-\sqrt{D_{E, i} \tau_{E, i}} \sin (f L+\phi) e^{2 L / \sqrt{D_{E, i} \tau_{E, i}}}-\sqrt{D_{E, i} \tau_{E, i}} \sin (f L+\phi)\right)}{\left(\frac{1+f_{i}^{2} \tau_{i} D_{E, i}}{A_{i}}\right)\left(-e^{2 L / \sqrt{D_{E, i} \tau_{E, i}}}+1\right)}, \\
& S_{i}=\frac{\left(D_{E, i} \cos \left(f_{i} L+\phi\right) f_{i} \tau_{i} e^{2 L / \sqrt{D_{E, i} \tau_{E, i}}}-D_{E, i} \cos \left(f_{i} L+\phi\right) f_{i} \tau_{i} e^{2 D / \sqrt{D_{E, i} \tau_{E, i}}}\right)}{\left(\frac{1+f_{i}^{2} \tau_{i} D_{E, i}}{A_{i}}\right)\left(-e^{2 L / \sqrt{D_{E, i} \tau_{E, i}}}+e^{\left.2 D / \sqrt{D_{E, i} \tau_{E, i}}\right)}\right.} \\
& +\frac{\left(2 \sqrt{D_{E, i} \tau_{E, i}} \sin \left(f_{i} D+\phi\right) e^{(D+L) / \sqrt{D_{E, i} \tau_{E, i}}}-\sqrt{D_{E, i} \tau_{E, i}} \sin (f L+\phi) e^{2 L / \sqrt{D_{E, i} \tau_{E, i}}}-\sqrt{D_{E, i} \tau_{E, i}} \sin (f L+\phi) e^{\left.2 D / \sqrt{D_{E, i} \tau_{E, i}}\right)}\right.}{\left(\frac{1+f_{i}^{2} \tau_{i} D_{E, i}}{A_{i}}\right)\left(-e^{2 L / \sqrt{D_{E, i} \tau_{E, i}}}+e^{2 D / \sqrt{D_{E, i} \tau_{E, i}}}\right)},
\end{aligned}
$$

To express the IPCE in terms of $S_{i}$, the charge separation yield at the photoactive interface into free charge carriers is assumed to be unity. Furthermore, it is assumed that all charge carriers formed on exciton dissociation are collected by the electrodes. The IPCE of the bilayer device then follows from

$$
\mathrm{IPCE}=\left(S_{\mathrm{P} 3 \mathrm{HT}}+S_{\mathrm{H}_{2} \mathrm{TMPyP}}\right) 100 \% .
$$

\section{APPENDIX D}

Figure 8 shows the atomic force micrographs of quartz, quartz/ $\mathrm{H}_{2} \mathrm{TMPyP}(25 \mathrm{~nm})$, and quartz/TiO ${ }_{2}$.

\section{APPENDIX E}

In the case of incoherent exciton hopping between molecules, the energy transfer rate $k_{\mathrm{ET}}$ between an energy donor and an energy acceptor depends on the excitonic coupling $V_{\mathrm{DA}}$ and the spectral overlap integral $J_{\mathrm{DA}}$ according to ${ }^{52,53}$

$$
k_{\mathrm{ET}}=\frac{2 \pi}{h}\left|V_{\mathrm{DA}}\right|^{2} J_{\mathrm{DA}} \text {. }
$$

The factor $J_{\mathrm{DA}}$ corresponds to the Franck-Condon weighted density of states and can be obtained from

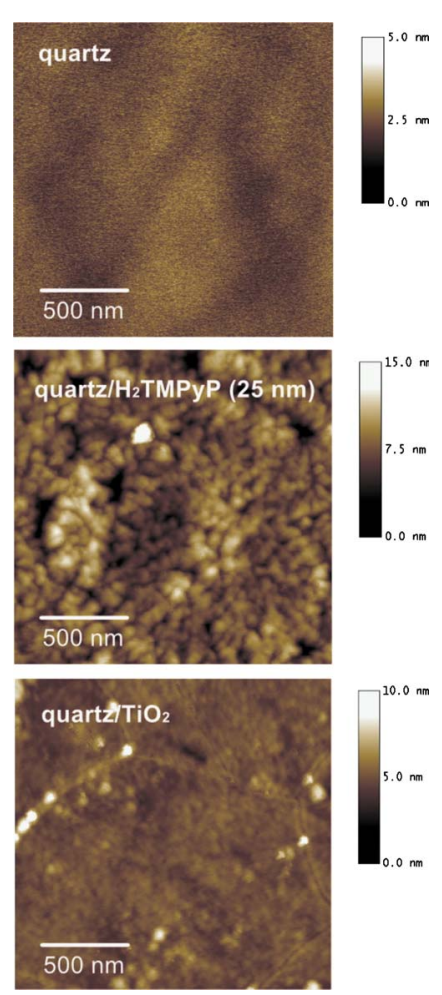

FIG. 8. (Color online) Atomic force micrographs of quartz, quartz/ $\mathrm{H}_{2} \mathrm{TMPyP}(25 \mathrm{~nm})$, and quartz/ $\mathrm{TiO}_{2}$. Note the different scales used. 

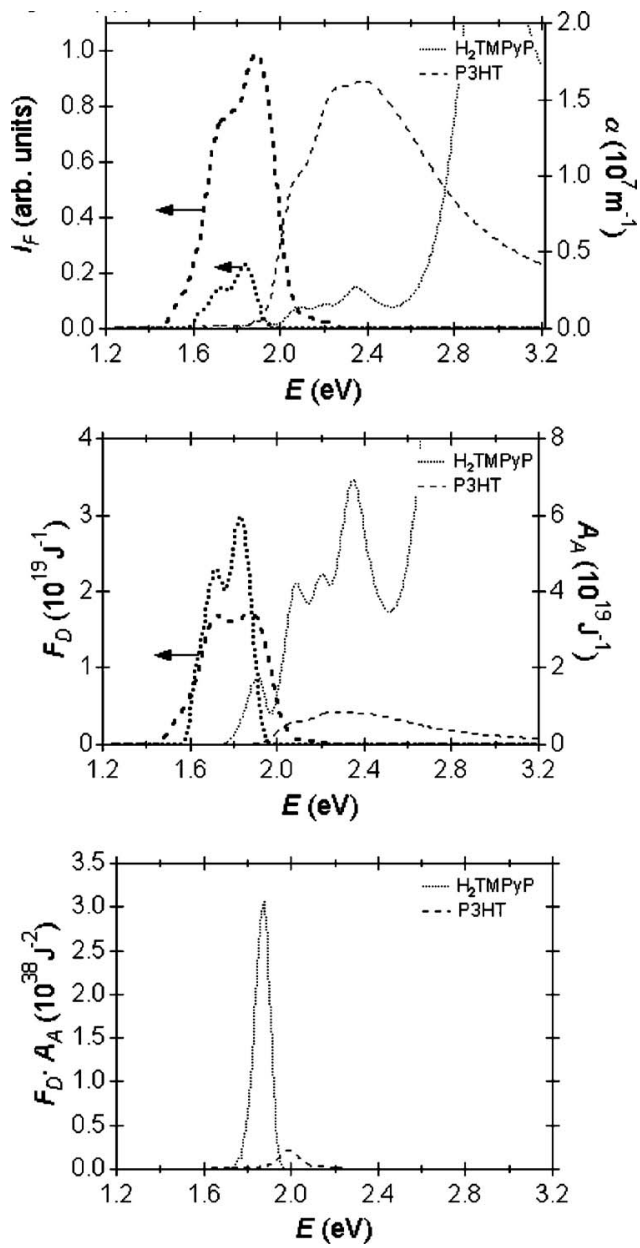

FIG. 9. Fluorescence and absorption of $\mathrm{H}_{2} \mathrm{TMPyP}$ and $\mathrm{P} 3 \mathrm{HT}$ as function of photon energy (top), fluorescence factor $F_{D}$ and absorption factor $F_{A}$ as function of photon energy (middle), and product of $F_{D}$ and $A_{A}$ as function of photon energy (bottom).

$$
J_{\mathrm{DA}}=\int F_{D}(E) A_{A}(E) d E,
$$

where $F_{D}$ and $A_{A}$ denote the emission factor of the energy donor and the absorption factor of the energy acceptor, respectively. ${ }^{52}$ The emission factor $F_{D}(E)$ depends on the fluorescence intensity $I_{F}(E)$ according to

$$
F_{D}(E)=\frac{E^{-3} I_{F}(E)}{\int E^{-3} I_{F}(E) d E} .
$$

The absorption factor $A_{A}(E)$ is related to the optical absorption coefficient $\alpha(E)$ by

$$
A_{A}(E)=\frac{E^{-1} \alpha(E)}{\int E^{-1} \alpha(E) d E} .
$$

Figure 9(a) shows the fluorescence intensity $I_{F}(E)$ and the optical absorption coefficient $\alpha(E)$ for $\mathrm{H}_{2}$ TMPyP and P3HT as function of photon energy $E$. The limits for integration of the functions shown in the denominators of Eqs. (E3) and (E4) are determined by the energy range of the transitions relevant for energy transfer. In the case of $\mathrm{H}_{2}$ TMPyP these limits follow from the energy range of the vibrational modes of the lowest $S_{0}-S_{1}$ electronic transition. For P3HT the functions shown in the denominator of Eqs. (E3) and (E4) are integrated over the entire $S_{0}-S_{1}$ absorption band. Application of Eqs. (E3) and (E4) then yields the emission factor $F_{D}(E)$ and the absorption factor $A_{A}(E)$, presented in Fig. 9(b). Multiplication of $F_{D}$ and $A_{A}$ for the individual compounds results in the curves presented in Fig. 9(c). Integration of these curves yields values for $J_{\mathrm{DA}}$ equal to 4.3 $\times 10^{18} \mathrm{~J}^{-1}$ for $\mathrm{H}_{2}$ TMPyP and $5.5 \times 10^{17} \mathrm{~J}^{-1}$ for P3HT.

${ }^{1}$ M. Knupfer, Appl. Phys. A: Mater. Sci. Process. 77, 623 (2003).

${ }^{2}$ I. G. Hill, A. Kahn, Z. G. Soos, and R. A. Pascal, Chem. Phys. Lett. 327, 181 (2000).

${ }^{3}$ J. W. van der Horst, P. A. Bobbert, M. A. J. Michels, G. Brocks, and P. J. Kelly, Synth. Met. 101, 333 (1999).

${ }^{4}$ K. Kajihara, K. Tanaka, K. Hirao, and N. Soga, Jpn. J. Appl. Phys., Part 1 36, 5537 (1997).

${ }^{5}$ T. J. Savenije, J. M. Warman, and A. Goossens, Chem. Phys. Lett. 287, 148 (1998)

${ }^{6}$ G. Horowitz, F. Kouki, P. Spearman, D. Fichou, C. Nogues, X. Pan, and F. Garnier, Adv. Mater. (Weinheim, Ger.) 8, 242 (1996).

${ }^{7}$ J. J. M. Halls, K. Pichler, R. H. Friend, S. C. Moratti, and A. B. Holmes, Appl. Phys. Lett. 68, 3120 (1996).

${ }^{8}$ J. E. Kroeze, T. J. Savenije, M. J. W. Vermeulen, and J. M. Warman, J. Phys. Chem. B 107, 7696 (2003).

${ }^{9}$ Y. X. Liu, M. A. Summers, C. Edder, J. M. J. Frechet, and M. D. McGehee, Adv. Mater. (Weinheim, Ger.) 17, 2960 (2005).

${ }^{10}$ D. E. Markov, E. Amsterdam, P. W. M. Blom, A. B. Sieval, and J. C. Hummelen, J. Phys. Chem. A 109, 5266 (2005).

${ }^{11}$ A. J. Lewis, A. Ruseckas, O. P. M. Gaudin, G. R. Webster, P. L. Burn, and I. D. W. Samuel, Org. Electron. 7, 452 (2006).

${ }^{12}$ Literature values for $\Lambda_{E}$ are multiplied by $\sqrt{2}$ to yield $\Lambda_{D}$.

${ }^{13}$ G. Yu, J. Gao, J. C. Hummelen, F. Wudl, and A. J. Heeger, Science 270, 1789 (1995).

${ }^{14}$ J. J. M. Halls, C. A. Walsh, N. C. Greenham, E. A. Marseglia, R. H. Friend, S. C. Moratti, and A. B. Holmes, Nature (London) 376, 498 (1995).

${ }^{15}$ G. Li, V. Shrotriya, J. Huang, Y. Yao, T. Moriarty, K. Emery, and Y. Yang, Nat. Mater. 4, 864 (2005).

${ }^{16}$ Y. Kim, S. Cook, S. M. Tuladhar, S. A. Choulis, J. Nelson, J. R. Durrant, D. D. C. Bradley, M. Giles, I. McCulloch, C. S. Ha, and M. Ree, Nat. Mater. 5, 197 (2006).

${ }^{17}$ V. Shrotriya, Y. Yao, G. Li, and Y. Yang, Appl. Phys. Lett. 89, 063505 (2006).

${ }^{18}$ H. Hoppe, M. Niggemann, C. Winder, J. Kraut, R. Hiesgen, A. Hinsch, D. Meissner, and N. S. Sariciftci, Adv. Funct. Mater. 14, 1005 (2004).

${ }^{19}$ C. Melzer, E. J. Koop, V. D. Mihailetchi, and P. W. M. Blom, Adv. Funct. Mater. 14, 865 (2004).

${ }^{20}$ J. K. J. van Duren, X. N. Yang, J. Loos, C. W. T. Bulle-Lieuwma, A. B. Sieval, J. C. Hummelen, and R. A. J. Janssen, Adv. Funct. Mater. 14, 425 (2004).

${ }^{21}$ D. Chirvase, J. Parisi, J. C. Hummelen, and V. Dyakonov, Nanotechnology 15, 1317 (2004).

${ }^{22}$ T. J. Savenije, J. E. Kroeze, X. N. Yang, and J. Loos, Adv. Funct. Mater. 15, 1260 (2005).

${ }^{23}$ H. J. Fell, E. J. Samuelsen, J. Alsnielsen, G. Grubel, and J. Mardalen, Solid State Commun. 94, 843 (1995).

${ }^{24}$ H. Sirringhaus, P. J. Brown, R. H. Friend, M. M. Nielsen, K. Bechgaard, B. M. W. Langeveld-Voss, A. J. H. Spiering, R. A. J. Janssen, E. W. Meijer, P. Herwig, and D. M. de Leeuw, Nature (London) 401, 685 (1999).

${ }^{25}$ K. E. Aasmundtveit, E. J. Samuelsen, M. Guldstein, C. Steinsland, O. Flornes, C. Fagermo, T. M. Seeberg, L. A. A. Pettersson, O. Inganas, R. Feidenhans'l, and S. Ferrer, Macromolecules 33, 3120 (2000).

${ }^{26}$ A. Zen, M. Saphiannikova, D. Neher, J. Grenzer, S. Grigorian, U. Pietsch, U. Asawapirom, S. Janietz, U. Scherf, I. Lieberwirth, and G. Wegner, Macromolecules 39, 2162 (2006).

${ }^{27}$ M. Brinkmann and P. Rannou, Adv. Funct. Mater. 17, 101 (2007).

${ }^{28}$ E. Moons, A. Goossens, and T. Savenije, J. Phys. Chem. B 101, 8492 (1997).

${ }^{29}$ T. J. Savenije, E. Moons, G. K. Boschloo, A. Goossens, and T. J. Schaaf- 
sma, Phys. Rev. B 55, 9685 (1997).

${ }^{30}$ C. W. Tang, Appl. Phys. Lett. 48, 183 (1986).

${ }^{31}$ P. Peumans and S. R. Forrest, Appl. Phys. Lett. 79, 126 (2001).

${ }^{32}$ P. Peumans, A. Yakimov, and S. R. Forrest, J. Appl. Phys. 93, 3693 (2003).

${ }^{33}$ P. Peumans, S. Uchida, and S. R. Forrest, Nature (London) 425, 158 (2003).

${ }^{34}$ T. J. Savenije, R. B. M. Koehorst, and T. J. Schaafsma, Chem. Phys. Lett. 244, 363 (1995).

${ }^{35}$ J. E. Kroeze, T. J. Savenije, and J. M. Warman, J. Am. Chem. Soc. 126 7608 (2004).

${ }^{36}$ S. M. Sze, Physics of Semiconductor Devices, 2nd ed. (Wiley, New York, 1981).

${ }^{37}$ L. A. A. Pettersson, L. S. Roman, and O. Inganas, J. Appl. Phys. 86, 487 (1999).

${ }^{38}$ M. Drees, R. M. Davis, and J. R. Heflin, J. Appl. Phys. 97, 036103 (2005).

${ }^{39}$ E. Itoh and K. Miyairi, Thin Solid Films 499, 95 (2006).

${ }^{40}$ M. A. Green, Solar Cells, Operating Principles, Technology, and System Applications (The University of New South Wales, Kensington, NSW, 1998).

${ }^{41}$ G. Horowitz, X. Peng, D. Fichou, and F. Garnier, J. Appl. Phys. 67, 528 (1990).

${ }^{42}$ D. M. Taylor, H. L. Gomes, A. E. Underhill, S. Edge, and P. I. Clemenson, J. Phys. D 24, 2032 (1991).
${ }^{43}$ M. S. A. Abdou, F. P. Orfino, Y. K. Son, and S. Holdcroft, J. Am. Chem. Soc. 119, 4518 (1997).

${ }^{44}$ S. Hoshino, M. Yoshida, S. Uemura, T. Kodzasa, N. Takada, T. Kamata, and K. Yase, J. Appl. Phys. 95, 5088 (2004).

${ }^{45}$ K. Yamashita, Y. Harima, and H. Iwashima, J. Phys. Chem. 91, 3055 (1987).

${ }^{46}$ W. Theiss, Dr.-Bernhard-Klein-Str. 110, D-52078 Aachen, Germany.

${ }^{47}$ O. Simpson, Proc. R. Soc. London, Ser. A 238, 402 (1957).

${ }^{48}$ M. Theander, A. Yartsev, D. Zigmantas, V. Sundstrom, W. Mammo, M. R. Andersson, and O. Inganas, Phys. Rev. B 61, 12957 (2000).

${ }^{49}$ K. R. Wu, J. J. Wang, W. C. Liu, Z. S. Chen, and J. K. Wu, Appl. Surf. Sci. 252, 5829 (2006).

${ }^{50}$ O. Mermut, D. C. Phillips, R. L. York, K. R. McCrea, R. S. Ward, and G. A. Somorjai, J. Am. Chem. Soc. 128, 3598 (2006).

${ }^{51}$ B. D. Vogt, C. L. Soles, H. J. Lee, E. K. Lin, and W. Wu, Polymer 46, 1635 (2005).

${ }^{52}$ M. Pope and C. E. Svenberg, Electronic Processes in Organic Crystals and Polymers, 2nd ed. (Oxford Science, New York, 1999).

${ }^{53}$ E. Hennebicq, G. Pourtois, G. D. Scholes, L. M. Herz, D. M. Russell, C. Silva, S. Setayesh, A. C. Grimsdale, K. Mullen, J. L. Bredas, and D. Beljonne, J. Am. Chem. Soc. 127, 4744 (2005).

${ }^{54}$ P. S. Tuminello, E. T. Arakawa, T. Inagaki, and J. E. Parks, J. Chem. Phys. 95, 4790 (1991) 